\title{
LOS DISPOSITIVOS MÓVILES EN LA ENSEÑANZA DE LA INVESTIGACIÓN EN INGENIERIA
}

\author{
Mobile devices in engineering research teaching
}

EPISTEMUS

ISSN: 2007-8196 (electrónico) ISSN: 2007-4530 (impresa)

Abelardo Mancinas González

Recibido: 14 de septiembre de 2017 Aceptado: 30 de mayo de 2018

Autor de Correspondencia: Dr. Abelardo mancinas González Correo:amancinas@ith.mx

\section{Resumen}

Por sus características de ubicuidad, interactividad y lenguaje multimedia los dispositivos móviles pueden ser utilizados como medios para enseñar la investigación y el diseño en el campo de la ingeniería. En este artículo se describe un estudio con enfoque mixto y diseño de estudio de caso, realizado con estudiantes de ingeniería en un curso de introducción a la investigación. Los alumnos utilizaron smarphones para capturar datos y crear información con fines de aprendizaje del proceso de investigación y diseño colaborativo de sistemas computacionales. Los resultados muestran que los dispositivos móviles no tuvieron un impacto en la capacidad de los estudiantes para analizar y formular problemas de investigación. Sin embargo, se evidencian factores como el grado de motivación para aprender con este tipo de herramientas. Se destacan las posibilidades que estos dispositivos ofrecen para trabajar en contextos cambiantes, así como su uso en la investigación y el diseño en ingeniería.

Palabras clave: aprendizaje móvil, dispositivos móviles, investigación y diseño

\section{Abstract}

Due to its characteristics of ubiquity, interactivity and multimedia language, mobile devices can be used to teach research and design in the field of engineering. This article describes a study with mixed approach and case study design, conducted with engineering students during an introductory course of research. The students used smartphones to capture data and create information to learn the research process and collaborative design of computer systems. The results show that the use of mobile devices does not affect the ability of students to analyze and formulate research problems. However, factors such as the degree of motivation to learn with this type of tools are clear. The possibilities that mobile devices offer to work in changing contexts stand out, as well as their use in research and engineering design.

Keywords: Mobile learning, mobile devices, research and design. 


\section{INTRODUCCIÓN}

En tanto que rama de las tecnologías de la información y la comunicación (TIC) en educación, el aprendizaje móvil se puede definir como el uso de dispositivos portátiles con fines de aprendizaje, en situaciones o contextos específicos y de manera colaborativa [1, 2].

Dadas sus características de movilidad, acceso a la consulta de información de forma ubicua, comunicación, colaboración y captura de datos del entorno, artefactos como el smartphone o la tableta electrónica, por mencionar dos de los más conocidos y utilizados, se convierten en herramientas con un gran potencial para investigar y diseñar por parte de los estudiantes de ingeniería.

Sin embargo, debido a su aparición relativamente reciente en el ámbito universitario, la utilización de este tipo de dispositivos portátiles con fines didácticos aún se encuentra limitada a su uso como dispensadores de contenido y herramientas de colaboración [3, 4]. Son pocos los estudios que reportan experiencias educativas con fines de investigación y diseño [5], así como de análisis y resolución de problemas [6]. En especial, en este último caso en dónde los resultados de investigación son contradictorios, ya que unos afirman que el aprendizaje móvil si contribuye al desarrollo de habilidades superiores de pensamiento necesarias a la resolución de problemas $[7,8]$, mientras otros concluyen que dichas habilidades son más bien superficiales $[9,10]$. Por lo que se requiere ampliar el número de experiencias orientadas a explorar las posibilidades del aprendizaje móvil en este aspecto.
Además del uso de los dispositivos móviles como medios de acceso a la información y de aprendizaje colaborativo, también, se requiere fomentar su aplicación como instrumentos de captura de datos y creación de información desde una perspectiva de colaboración [11]. En el caso que aquí se presenta se pone el énfasis en la creación de información y diseño colaborativo de una aplicación de software para dispositivos móviles (app), mediante la utilización de smartphones, por parte de estudiantes de ingeniería en sistemas computacionales del Instituto Tecnológico de Hermosillo.

El objetivo del presente estudio consiste en explorar las posibilidades de los dispositivos móviles cómo herramientas de apoyo en la enseñanza de la investigación y diseño en el dominio de la ingeniería. La pregunta de investigación que lo guía: ¿Cuál es el rol de los dispositivos móviles como apoyo en la enseñanza de la investigación y el diseño colaborativo en ingeniería?

\section{MÉTODO}

La metodología utilizada es de tipo mixto con predominancia cualitativa y diseño de estudio de caso. Se aplicaron dos encuestas, al inicio y al cierre del curso,

a un total de 34 estudiantes. La primera con el fin de obtener datos acerca del uso y percepción de los dispositivos móviles de parte de los alumnos. La segunda, con el propósito de evaluar la percepción de estos últimos en relación con la experiencia de aprendizaje móvil.

Los tipos de usos, en tanto que categorías generales de análisis en la fase cuantitativa del estudio, fueron

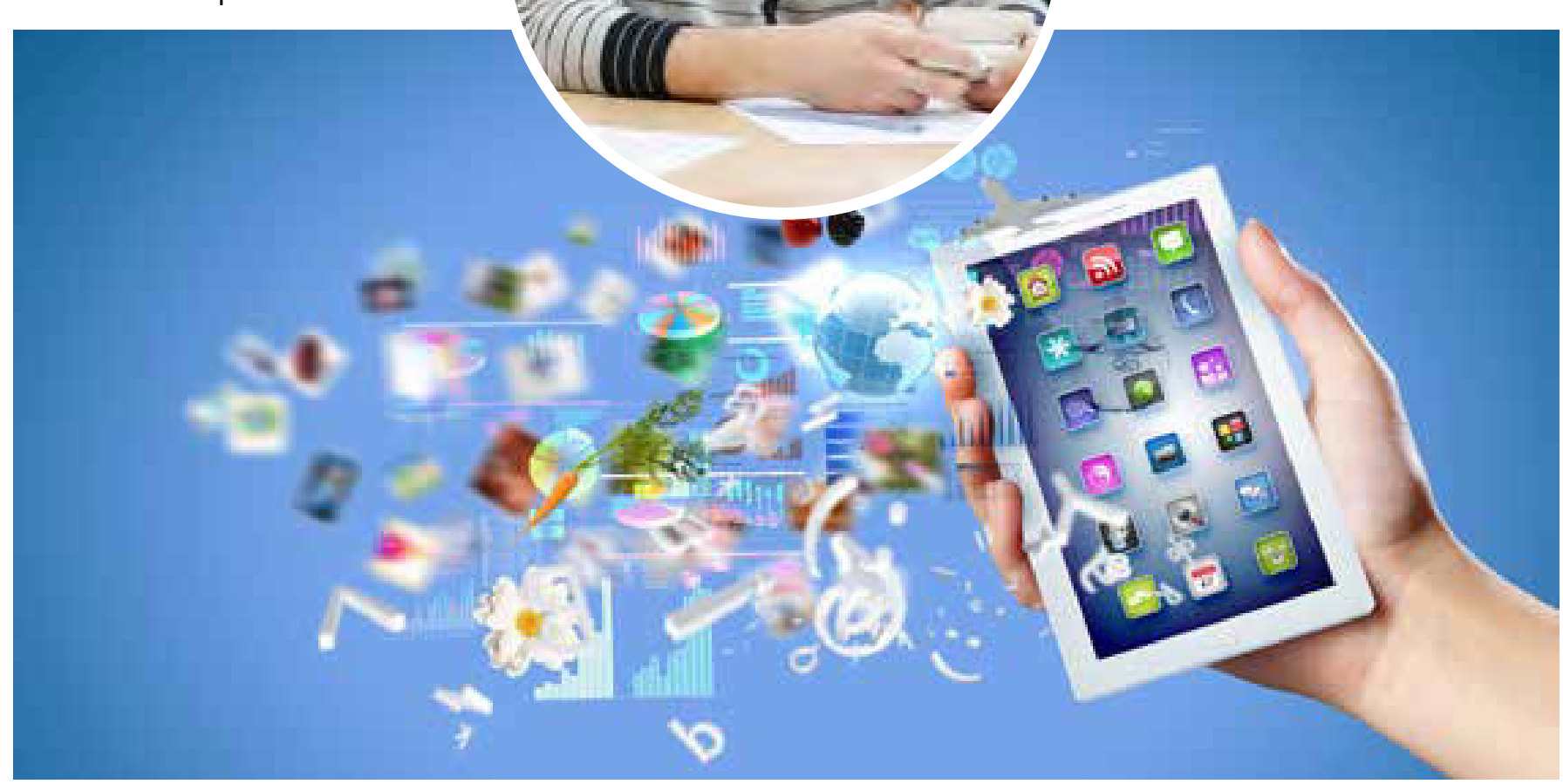




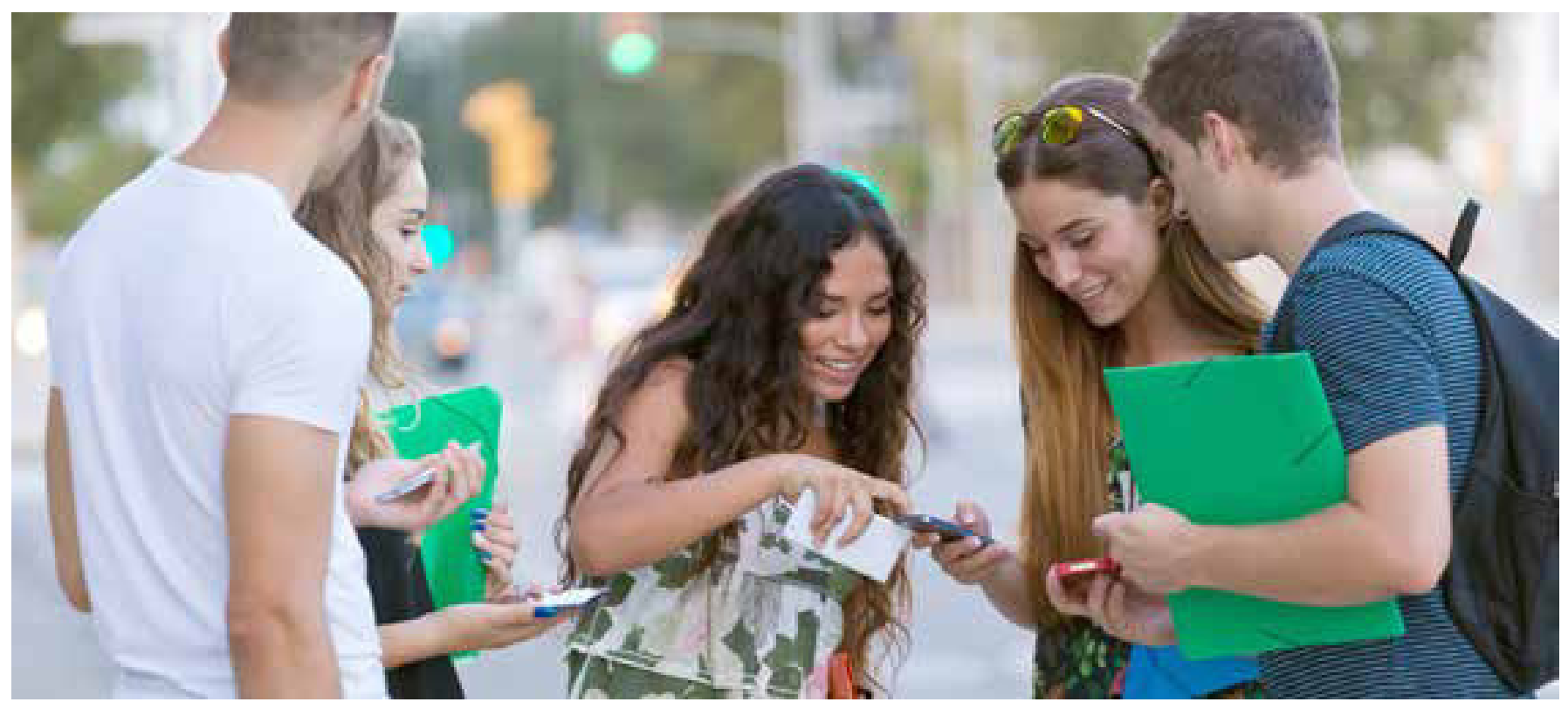

agrupados en función de los resultados obtenidos en otras investigaciones sobre la utilización de los samartphones por universitarios $[12,13]$.

También, durante y al término de la asignatura, se realizaron entrevistas semiestructuradas a los equipos de trabajo, con el objetivo de conocer el impacto en el aprendizaje logrado. Así mismo, se llevó el registro de la experiencia a través de una bitácora de campo.

La población del caso estuvo compuesta por un grupo de 34 alumnos, organizado en equipos de trabajo con un promedio de tres integrantes cada uno. Los estudiantes pertenecían al primer semestre de la carrera de Ingeniería en Sistemas Computacionales y cursaban la asignatura de Fundamentos de Investigación, semestre agostodiciembre de 2015.

El objetivo pedagógico de la asignatura consistió en diseñar, en equipo, una app en tanto que sistema de información que diera solución a una problemática del entorno de los estudiantes, apoyándose en el uso intensivo de dispositivos móviles. El proyecto se plasmó en un protocolo de investigación que incluyó el diseño de un prototipo, además del algoritmo correspondiente.

A cada equipo se le solicitó crear un proyecto a partir de una problemática específica del entorno, cuya solución pudiera ser abordada a través del diseño de una app. Fue así como surgieron proyectos de diseño de apps orientadas a disminuir los tiempos de espera de autobuses urbanos, el control del tráfico, la reservación de citas en instituciones de salud, controles de seguridad en casas habitación o asistentes personales para combinar la vestimenta de una persona.

Para identificar las necesidades específicas de los usuarios, los equipos se apoyaron en la suite de Kobo
Toolbox. Esta herramienta es utilizada por investigadores profesionales, en el campo de las ciencias sociales, para el diseño de cuestionarios, recolección y análisis de datos mediante el uso de smartphones.

La información resultante del estudio realizado por cada equipo de trabajo se presentó en un informe de resultados que, a su vez, sirvió de base para que los alumnos plantearan el problema y formularan la pregunta de investigación. A continuación, apoyados en sus teléfonos y la app de Evernote, la cual fue utilizada como una página Wiki, se solicitó a los equipos la elaboración de un marco conceptual que les ampliara la comprensión teórica del problema a abordar.

El siguiente paso consistió en la búsqueda de posibles soluciones a la problemática abordada, por lo cual se procedió al diseño de la app mediante la elaboración del viaje de usuario (user journey), definición de funciones, arquitectura de información y elaboración del primer bosquejo gráfico (wireframe) de las pantallas. Se continuó con el diseño del algoritmo en sus versiones de diagrama de flujo y pseudocódigo. En esta fase del proyecto los alumnos se apoyaron en aplicaciones en línea como LucidChart, la cual les permitió trabajar de manera colaborativa.

Finalmente, se procedió a la elaboración del prototipo de la app con el apoyo de software en línea como Fluid y Justinmind Prototyper. Además, mediante el recurso a los smartphones, los estudiantes realizaron pruebas de usabilidad a través de grabaciones de video y entrevistas semiestructuradas a los usuarios.

Junto con el diseño de la app los equipos entregaron un protocolo de investigación en el cual, además de la problemática, plasmaron un marco conceptual, definieron una metodología y avanzaron posibles conclusiones. Así 
docentes de Instituto para colocar materiales de los cursos presenciales y aplicar exámenes en línea. También, figura la descarga de música y videos como uno de los principales usos informales de este tipo de dispositivos. Así mismo, su utilización en el trabajo y la escuela se consideran como igualmente importantes.

Según manifestaron los encuestados, la creación de información mediante dispositivos móviles se limita a la elaboración de mensajes de texto, toma de fotografías y grabación audio y video para la creación de mensajes en las redes sociales.

En lo que concierne a la experiencia de trabajar con dispositivos móviles para realizar las actividades del curso, los estudiantes expresaron que tareas como la elaboración y aplicación de encuestas con el apoyo de apps les resultó fácil y práctica, al igual que el procesamiento de los datos y el análisis de los resultados. Sin embargo, no se observó ningún impacto derivado del uso de dispositivos móviles en el aprendizaje del análisis de datos y el planteamiento del problema. El impacto se ubica más bien en la motivación de los estudiantes para realizar las tareas con la ayuda de un smartphone.

El trabajo colaborativo a través de Everenote para elaborar el marco conceptual de la investigación tuvo dificultades para concretarse, ya que los integrantes de los equipos carecían de la experiencia necesaria para trabajar en la redacción colectiva de un texto apoyándose en una Wiki. El resultado fue una serie de textos, con frecuencia copiados de páginas Web, que no guardaban coherencia entre sí.

El diseño de la aplicación, en tanto que posible solución al problema previamente planteado, se llevó a cabo sin mayores dificultades. La totalidad de los participantes manifestó su entusiasmo e interés por realizar actividades que impliquen el diseño visual, tales como esquemas (user journey), diagramas de flujo (arquitectura de información) y bocetos (wireframes), todos ellos utilizados en el diseño de apps.

En esta última fase resalta la habilidad y el entusiasmo mostrado por los alumnos para traducir la solución del problema en cuestión en un algoritmo, así como en

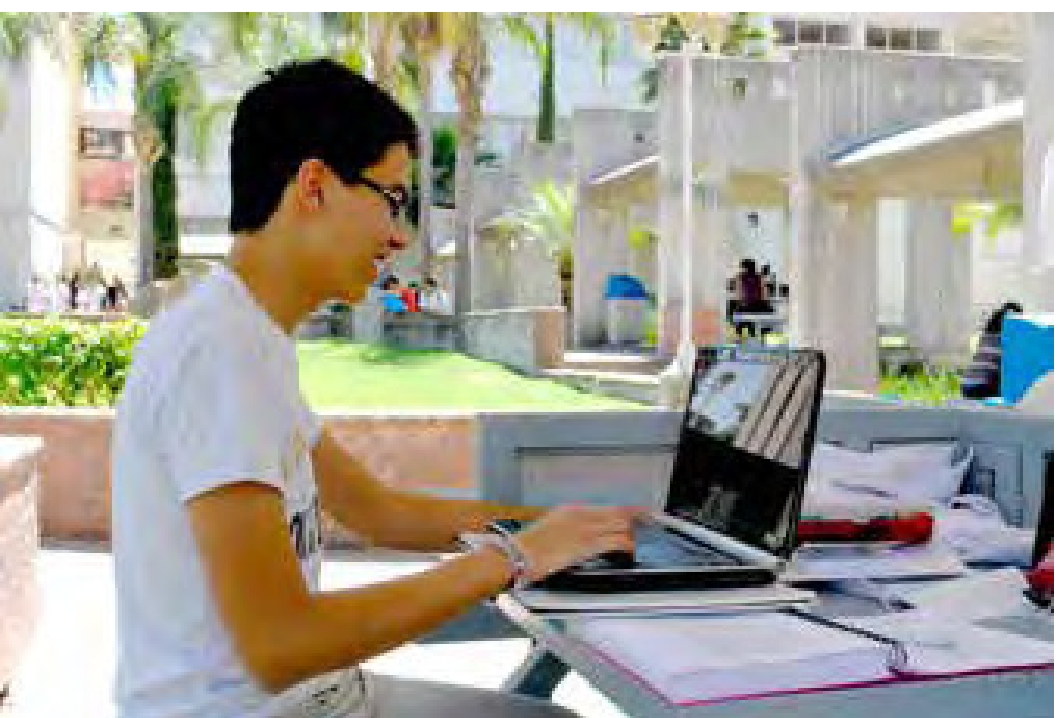

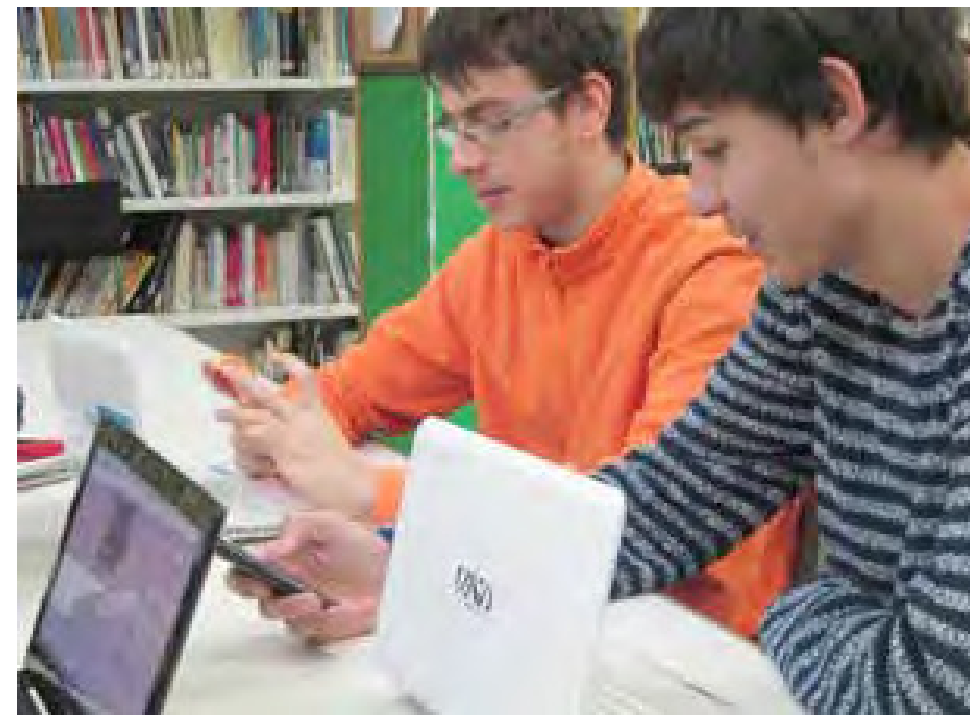

lenguaje gráfico y áptico propio de una aplicación de software para dispositivos móviles. Algo similar sucedió con la creación del prototipo en línea, elaborado mediante aplicaciones como Fluid y Justinmind Prototyper, el cual no presentó dificultades para los participantes.

Las pruebas de usabilidad, apoyadas en una entrevista semiestructurada y la grabación de video, permitieron a los estudiantes obtener retroalimentación de los usuarios y realizar los ajustes necesarios al prototipo antes de su entrega al final del semestre.

\section{DISCUSIÓN}

Como ya lo han señalado numerosos autores, la disposición de los alumnos hacia el uso de tecnología móvil en el aula es favorable, independientemente de la desconfianza y, en ocasiones, el rechazo abierto que suelen manifestar los docentes hacia esta forma de aprendizaje [14]. Por lo que la percepción positiva de los jóvenes hacia esta modalidad de educativa se expresa claramente en su motivación para usarla como medio de estudio. Lo antes expresado queda confirmado por las entrevistas, así como por investigadores en el dominio del aprendizaje móvil $[12,13]$.

En lo que concierne al diseño de instrumentos de captura de datos, como es el caso de cuestionarios $y$ entrevistas semiestructuradas para diagnosticar necesidades de los usuarios y elaborar pruebas de usabilidad, los alumnos mostraron un buen desempeño. La dificultad se presentó en el momento de organizar y presentar los resultados de la encuesta, así como en el planteamiento del problema, ya que no se observó una mejora con el uso de dispositivos móviles si se le compara con la utilización de otros medios como la computadora o el papel y lápiz.

Independientemente de que el docente de la asignatura recurrió a la estrategia didáctica de aprendizaje por proyectos, además de que proporcionó un seguimiento 


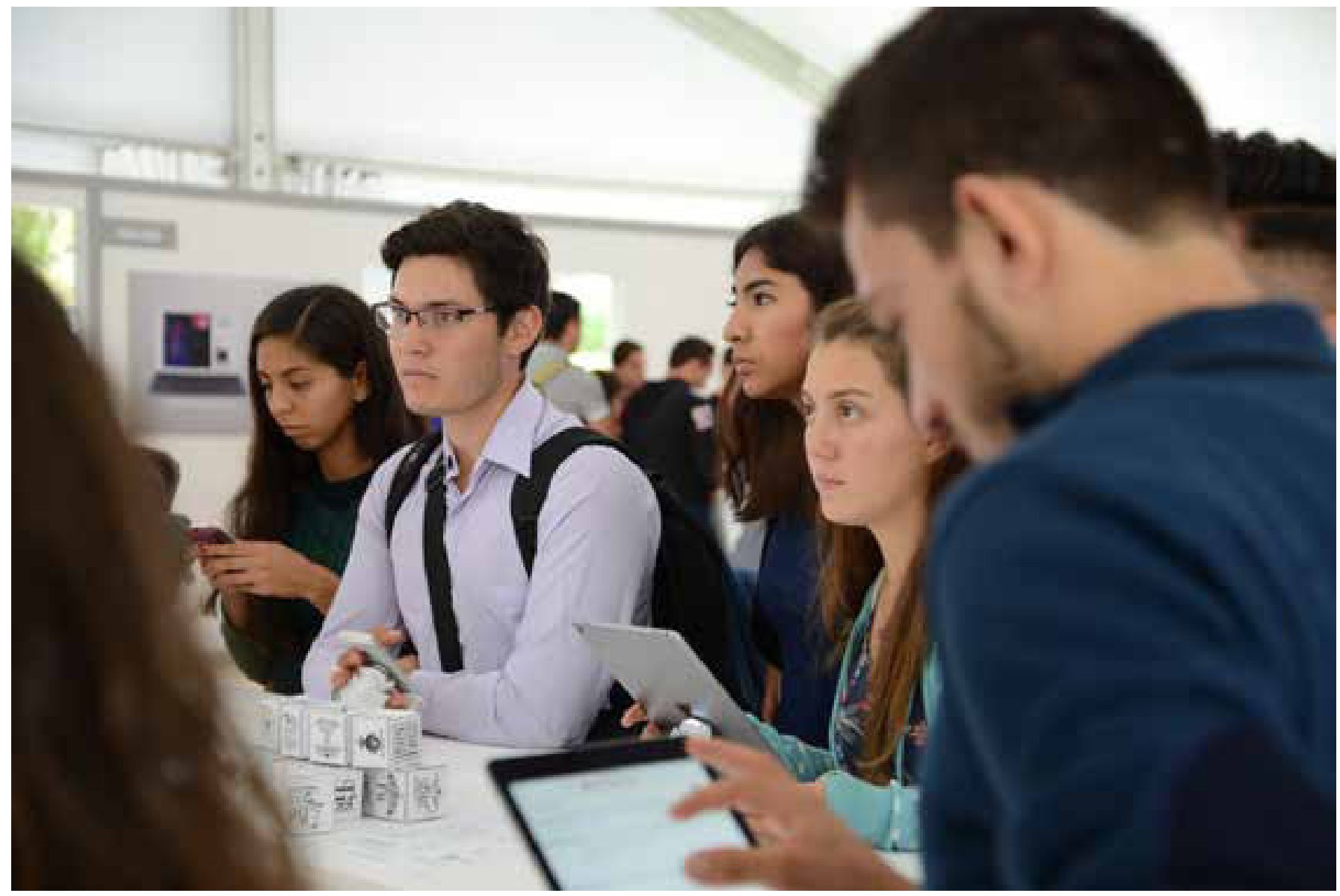

personalizado a los equipos de trabajo a través de sesiones de discusión de los avances de cada proyecto, este resultado se explica en parte debido dos factores: las deficiencias en el desarrollo de habilidades de análisis y pensamiento crítico que arrastran los estudiantes procedentes del nivel de bachillerato; así como el tipo de cognición, basada en heurísticas más que en un pensamiento reflexivo, que parece propiciar el uso de dispositivos móviles con fines de aprendizaje [15].

También, la colaboración entendida como interdependencia de los miembros de un equipo de trabajo no se logró. Los jóvenes tienden a trabajar en grupo de manera fragmentada. Aportan retazos de información de manera individual y no consideran lo redactado por sus compañeros, algo muy diferente de lo solicitado para realizar un ejercicio de escritura colaborativa en una página Wiki.

\section{CONCLUSIONES}

El objetivo del presente estudio consistió en explorar el rol de los dispositivos móviles cómo herramientas de apoyo en la enseñanza de la investigación y diseño en el dominio de la ingeniería. De la misma manera, la pregunta de investigación ha cuestionado acerca del rol de los dispositivos móviles como apoyo en la enseñanza de la

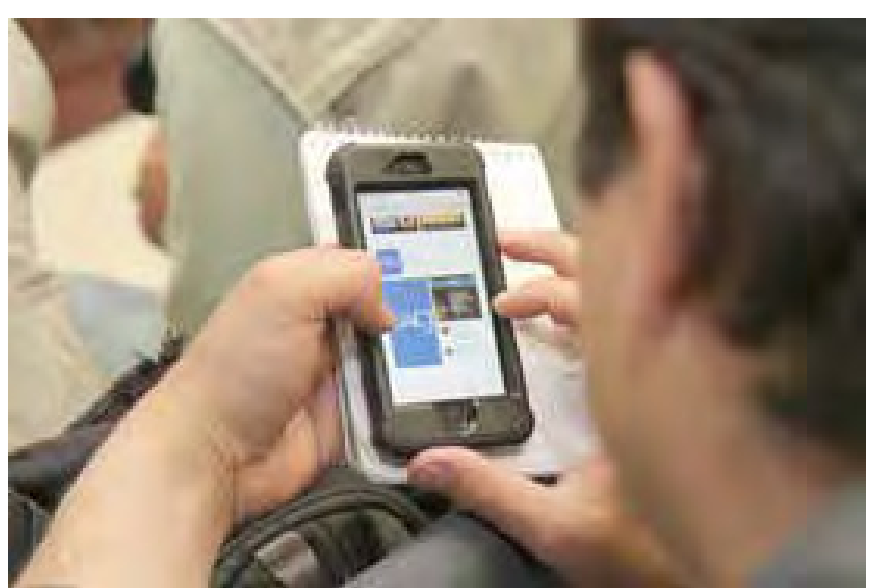

investigación y el diseño colaborativo en ingeniería.

En ese sentido se puede afirmar, en consonancia con otras investigaciones sobre el impacto del aprendizaje móvil en el desarrollo de habilidades superiores de pensamiento necesarias a la formulación y solución de problemas $[9,10,16]$, aspecto fundamental de todo proceso de investigación, que los dispositivos móviles no tienen un rol relevante en el desarrollo de capacidades del análisis, evaluación y toma de decisiones o en el fomento del pensamiento crítico y creativo en general. En cuanto 
al diseño, el aprendizaje móvil si tiene un papel favorable al favorecer la colaboración y la interacción entre los estudiantes en todo momento durante el desarrollo del proyecto

Sin embargo, en la medida en que dispositivos como el smartphone sean utilizados como herramientas de creación de información y conocimiento, sin limitar su utilización a la consulta y comunicación de datos, estos pueden tener un rol favorable en la enseñanza de la investigación.

Además, la posibilidad que experimentaron los alumnos de trabajar fuera del aula y la escuela en contextos significativos para ellos, constituyó una de las mejores experiencias según lo comentan. El aprendizaje situado, por lo tanto, constituye una oportunidad para el aprendizaje móvil como lo reconoce la UNESCO [17].

El proceso seguido para problematizar las necesidades de los usuarios y que desemboca en el diseño de la app, como la posible solución al problema planteado, muestra que estas herramientas tienen un potencial en la investigación y el diseño.

\section{BIBLIOGRAFÍA}

[1] C. Castaño, J. Cabero, Enseñar y aprender en entornos de M-Learning, Madrid: Síntesis, 2013.

[2] C. Daughtery, Z. Berge, "Mobile Learning Pedagogy", International Journal for the Scholarship of Technology Enhanced Learning, vol.1, no.2, pp. 111-118, 2017.

[3] M. Ally, A. Tsinakos, Increasing access through mobile learning, Vancouver: Commonwealth of Learning Press, 2014.

[4] B. Chen, R. Seilhamer, L. Bennett, S. Bauer,"Students' Mobile Learning Practices in Higher Education: A Multi-Year Study", EDUCASE Review, Jun. 2015. [Online]. Available: http:// er.educause.edu/articles/2015/6/students-mobile-learningpractices-in-higher-education-a-multiyear-study

[5] Y.-T. Sung, K.-E. Chang, T.Ch. Liu, "The effects of integrating mobile devices with teaching and learning on students' learning performance: A meta-analysis and research synthesis", Computers \& Education, vol. 9, pp. 252-275, 2016.

[6] M. Fuad, D. Deb, S.Whitaker, "Mobile Interactive Problem Solving for Active Teaching and Learning", in Proc. 8th International Conference on Electrical and Computer Engineering, Dhaka, Bangladesh, 2014. [Online]. Available: https://par.nsf.gov/servlets/purl/10017487

[7] G. Chávez Saavedra, B.V. González Sandoval, C. Hidalgo Valadez, "Aprendizaje Basado en Problemas (ABP) a través del m-learning para el abordaje de casos clínicos. Una propuesta innovadora en educación médica", Innovación Educativa, vol. 16, no.72, pp. 95-112, 2016.

[8] G.-J. Hwang, H.-F. Chang, "A formative assessment-based mobile learning approach to improving the learning attitudes and achievements of students", Computers \& Education, no. 56, pp.1023-1031, 2011.

[9] L. Vargas Mendoza, M.G. Gómez Zermeño, R.L. Gómez Zermeño, "Desarrollo de habilidades cognitivas y tecnológicas con aprendizaje móvil", Revista de Investigación Educativa de la Escuela de Graduados en Educación, no. 6, pp. 30-39, 2013.

[10] J.M. Zydney, Z. Warner, "Mobile apps for science learning: Review of research". Computers \& Education, no. 94, pp. 1-17, 2016.

[11] Ch.Yin, "SAMCCO: un Système d'Apprentissage Mobile
Contextuel et Collaboratif dans des Situations Professionnelles", Ph.D. dissertation, Ecole Centrale de Lyon, Lyon, France, february 2011

[12] J. Organista-Sandoval, A. Serrano-Santoyo, L. McAnallySalas, G. Lavigne, "Apropiación y usos educativos del celular por estudiantes universitarios", Revista Electrónica de Investigación Educativa, vol.15, no.3, pp.138 -156, 2013.

[13] S. W. Tabor, "Making mobile learning work: Student perceptions and implementation factors", Journal of Information Technology Education: Innovations in Practice, vol.15, pp. 75-98, 2016.

[14] C. Shuler, N. Winters, M. West, "El futuro del aprendizaje móvil. Implicaciones para la planificación y formulación de políticas", UNESCO, Paris, UNESCO Report, 2013. Available: http://unesdoc.unesco.org/images/0021/002196/219637s. pdf

[15] M. Serres, Petite Poucette, Paris: Le Pommier, 2012.

[16] J.C. Yen, C.Y. Lee, "Exploring problem solving patterns and their impact on learning achievement in a blended learning environment, Computers \& Education, no. 56, pp.138-145, 2011.

[17] M. West, "Activando el aprendizaje móvil: temas globales", UNESCO, París, UNESCO Report 2012. Available: unesdoc. unesco.org/images/0021/002164/216451s.pdf

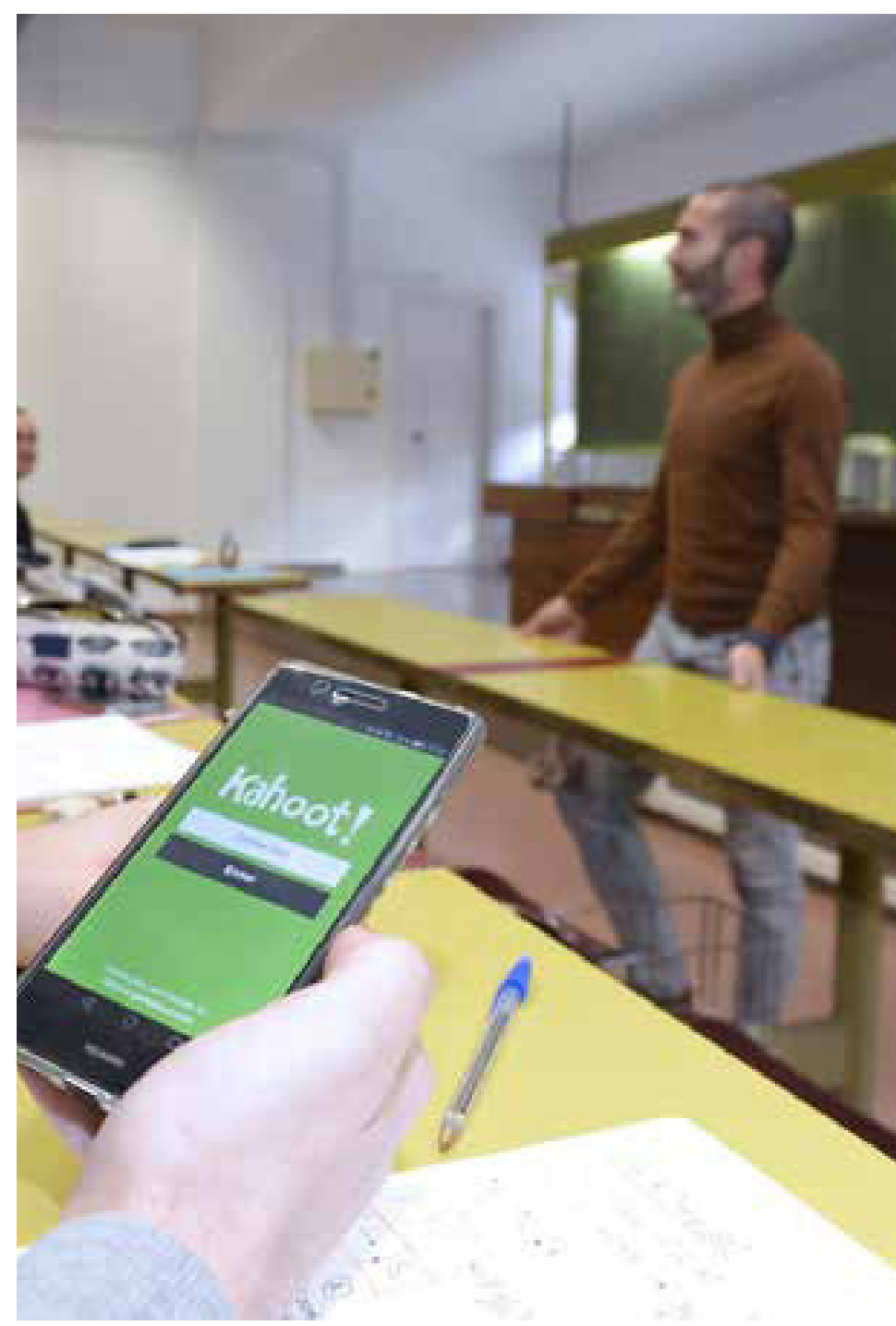

